\title{
Olfactory Dysfunction: A Potentially Negative Sign for Depression
}

\author{
Zhengwei Wen ${ }^{1 \#}$, Hua-Zhen Lin ${ }^{2,3 \#}$, Yao-Yao Li ${ }^{4,5 H}$ and Yun-Feng Zhang ${ }^{4,5,6^{*}}$
}

${ }^{1}$ The First Affiliated Hospital, Wenzhou Medical University, China

${ }^{2}$ The Second Affiliated Hospital and Yuying Children's Hospital, Wenzhou Medical University, China

${ }^{3}$ The 2nd School of Medicine, Wenzhou Medical University, China

${ }^{4}$ School of Ophthalmology \& Optometry, The Eye Hospital, Wenzhou Medical University, China

${ }^{5}$ State Key Laboratory Cultivation Base and Key Laboratory of Vision Science, Ministry of Health P. R. China and Zhejiang Provincial Key Laboratory of Ophthalmology and Optometry, China

${ }^{6}$ Department of Neuroscience, University of Pennsylvania Perelman School of Medicine, USA

${ }^{\#}$ Equal contribution.

*Corresponding author: Yun-Feng Zhang, Department of Neuroscience, University of Pennsylvania Perelman School of Medicine, Philadelphia, PA, USA, E-mail: zhangyunfeng1983@163.com

\begin{abstract}
Depression is a typical neuropsychiatric disorder and has led to great economic and social burden worldwide. During the past few decades, though great efforts have been made on parsing out this mental disease, the potential association of olfactory dysfunctions with depression still remains much to be characterized. Here in this short review, we concisely summarized the evidence of how olfactory dysfunctions acting as a negative marker potentially sign the depression. The olfactory-bulbecomized $(\mathrm{OB})$ rat model highlights the potentially crucial role of olfaction in the pathophysiology of depression. Multiple clinical studies have showed that patients with dysfunctions in olfactory system exhibit a higher probability of depression. Moreover, nowadays the olfaction is considered as a useful approach in the therapy of depression, and olfactory stimulation can potentially ameliorate depressive symptoms. Olfactory dysfunctions, at least in part, are resulted from the decreased olfactory input which can influence neurotransmitter release and potentially changes functioning in limbic and reward related brain areas. The decrease or totally loss of olfactory input may lead to the imbalance of neuronal emotion processing and thereby results in the depressive symptoms in people.
\end{abstract}

\section{Text}

Depression is a typical neuropsychiatric disorder characterized by anhedonia [1,2] which is considered to be the fourth leading cause of disability and will become the second by $2020[3,4]$. Depression has produced great burden to both the economy and society worldwide. During the past few years, though great efforts have been made on parsing out this neurological disorder [4-12], which are accompanied with a plethora of animal models of depression including the olfactory-bulbecomized rat model emerged aiming to unveil the underlying mechanism [4,5,13-15], the association of olfactory dysfunctions with depression still remains much to be characterized. Among these models, the olfactory-bulbecomized (OB) rat model highlights the potentially crucial role of olfaction in the pathophysiology of depression. In the current short review, for concise we only briefly summarized the evidence of how olfactory dysfunction acting as a negative marker potentially signs the depression.

Historic study has suggested that the $O B$ rat is not only a means for testing putative antidepressants but also is a model of depression [14,16]. A growing body of evidence reveals the negative correlation between the olfactory function and depressive symptoms [17-23]. Olfactory dysfunction not only decreases the quality of life but also weakens function and activities of daily living. Clinical studies have showed that patients with dysfunctions in olfactory system exhibited a higher probability of depressive-like phenotypes [24-26], further suggesting olfaction as a negative marker for depression.

This fact is further supported by a recent study indicating that poor odor identification is closely asso- 
ciated with increased depressive symptoms in older male adults [27]. More interestingly, nowadays olfactory dysfunction has been used as a screen measure to discriminate major depression from other neurological disorders such as Alzheimer's disease $[28,29]$, and also the olfactory bulb volume is used to predict the therapeutic outcome of major depression disorder, at least in women patients [30]. In addition, the olfaction is also considered as a useful approach in the therapy of depression, and olfactory stimulation can potentially ameliorate depressive symptoms [18]. The clinical evidence confirmed that performing olfactory training using the Sniffin' Sticks test led to improved olfactory function in patients with olfactory loss [31], which in further could weaken the severity of depressive symptoms in older patients [32]. It was documented that deep brain stimulation or non-invasive brain stimulation targeting the olfactory system produced "immediate relief" on major depression patients [33,34], which has provided new therapeutic opportunities for treatment on patients with depression. Taken together, olfactory dysfunction is a potentially negative marker for depression, and focusing on the improvement of olfactory function in patients with depression could be worthy of considering as a useful measure in ameliorating the depressive symptoms.

The olfactory bulbectomy could result in remarkable modifications in various brain areas, which may be, at least in part, due to the abnormal connections between the olfactory bulb and other brain regions, mainly the olfactory-limbic circuitry [14]. Olfactory dysfunctions such as the decreased volume or destroy of the olfactory bulb may decrease the olfactory input to the limbic system [30]. Some brain areas involved in emotion processing such as amygdaloid nuclei, anterior cingulate cortex, insular regions and orbitofrontal cortex all received strong projections from the olfactory bulb [35]. The decreased olfactory input could influence neurotransmitter release and potentially changed functioning in limbic and reward related brain areas [18]. A decrease or totally loss of olfactory input would also possibly result in the imbalance of neuronal emotion processing and thereby leads to the depressive symptoms in people.

Olfactory dysfunctions in depression patients could happen at both the peripheral and central processing levels. Basically, the peripheral olfactory processing involves the acuity, detection, and sensitivity of olfaction, all of which is evaluated using measures of the olfactory threshold $[23,36,37]$. Dysfunctions in the peripheral processing are reflected by the weakened processing ability at the level of the nasal epithelium. The central olfactory processing is generally reflected by the identification and discrimination of an odor [23,36-38]. Olfactory processing at these two different levels opens the question that what is the weight of abnormality in peripheral and central olfactory processing, respective-
Iy, in the pathogenesis of depression. It is worthy clarifying the potentially different roles of the peripheral and central olfactory system abnormalities in the depressive symptoms in the future studies.

\section{References}

1. Willner P, Muscat R, Papp M (1992) Chronic mild stress-induced anhedonia: A realistic animal model of depression. Neurosci Biobehav Rev 16: 525-534.

2. Keedwell PA, Andrew C, Williams SC, Brammer MJ, Phillips ML (2005) The neural correlates of anhedonia in major depressive disorder. Biol Psychiatry 58: 843-853.

3. Bromet E, Andrade LH, Hwang I, Sampson NA, Alonso J, et al. (2011) Cross-national epidemiology of DSM-IV major depressive episode. BMC Med 9: 90.

4. Lu C, Wang Y, Zhang YF (2016) Light deprivation produces a sexual dimorphic effect on neural excitability and depression-like behavior in mice. Neurosci Lett 633: 69-76.

5. Zhang YF, Li QQ, Qu J, Sun CM, Wang Y (2016) Alterations of motor cortical microcircuit in a depressive-like mouse model produced by light deprivation. Neuroscience 79-94.

6. Gonzalez MM, Aston-Jones G (2008) Light deprivation damages monoamine neurons and produces a depressive behavioral phenotype in rats. Proc Natl Acad Sci U S A 105: 4898-4903.

7. Zhu X, Li R, Wang P, Li J (2014) Aberrant functional connectivity of the hippocampus in older adults with subthreshold depression. Psych J 3: 245-253

8. Li YY, W ZW, Hu SS, Zhang YF (2017) The role of lateral habenula in depression: What we know and what we don't yet know. Neurol Sci J 1: 10.

9. Chen YC, Bo F, Xia W, Liu S, Wang P, et al. (2017) Amygdala functional disconnection with the prefrontal-cingulate-temporal circuit in chronic tinnitus patients with depressive mood. Prog Neuropsychopharmacol Biol Psychiatry 79: $249-257$

10. Lu C, L YY, Lin HZ, Zhang YF (2017) Depression: Abnormality in Neural Circuits. Annals of Psychiatry and Mental Health 5: 1115.

11. Lu C, Lin HZ, L YY, Zhang YF (2017) Making room for specific molecules in the treatment of depression. Scientific Journal of Depression \& Anxiety 1: 20-22.

12. Knowland D, Lilascharoen V, Pacia CP, Shin S, Wang EH, et al. (2017) Distinct ventral pallidal neural populations mediate separate symptoms of depression. Cell 170: 284-297.

13. Duman $\mathrm{CH}$ (2010) Models of depression. Vitam Horm 82: $1-21$.

14. Song C, Leonard BE (2005) The olfactory bulbectomised rat as a model of depression. Neurosci Biobehav Rev 29: 627-647.

15. Lu C, Lin HZ, Li YY, Zhang YF (2017) Light deprivation: An efficient way in inducing depression-like behavior animal models. Res Rev Insights 1: 1-3.

16. Kelly JP, Wrynn AS, Leonard BE (1997) The olfactory bulbectomized rat as a model of depression: An update. Pharmacol Ther 74: 299-316.

17. Wen ZW, Lu C, Lin HZ, Li YY, Zhang YF (2017) A short glance at the role of olfaction in depression. Arch Depress Anxiety $3: 55-57$. 
18. Croy I, Hummel T (2017) Olfaction as a marker for depression. J Neurol 264: 631-638.

19. Yuan TF, Slotnick BM (2014) Roles of olfactory system dysfunction in depression. Prog Neuropsychopharmacol Biol Psychiatry 54: 26-30.

20. Croy I, Symmank A, Schellong J, Hummel C, Gerber J, et al. (2014) Olfaction as a marker for depression in humans. J Affect Disord 160: 80-86.

21. Pollatos O, Albrecht J, Kopietz R, Linn J, Schoepf V, et al. (2007) Reduced olfactory sensitivity in subjects with depressive symptoms. J Affect Disorders 102: 101-108.

22. Pause BM, Miranda A, Goder R, Aldenhoff JB, Ferstl R (2001) Reduced olfactory performance in patients with major depression. J Psychiatr Res 35: 271-277.

23. Negoias S, Croy I, Gerber J, Puschmann S, Petrowski K, et al. (2010) Reduced olfactory bulb volume and olfactory sensitivity in patients with acute major depression. Neuroscience 169: 415-421.

24. Temmel AF, Quint C, Schickinger-Fischer B, Klimek L, Stoller E, et al. (2002) Characteristics of olfactory disorders in relation to major causes of olfactory loss. Arch Otolaryngol Head Neck Surg 128: 635-641.

25. Croy I, Landis BN, Meusel T, Seo H-S, Krone F, et al. (2011) Patient adjustment to reduced olfactory function. Arch Otolaryngol Head Neck Surg 137: 377-382.

26. Croy I, Hummel T, Pade A, Pade J (2010) Quality of life following nasal surgery. Laryngoscope 120: 826-831.

27. Sivam A, Wroblewski KE, Alkorta-Aranburu G, Barnes LL, Wilson RS, et al. (2016) Olfactory dysfunction in older adults is associated with feelings of depression and loneliness. Chem Senses 41: 293-299.

28. McCaffrey RJ, Duff K, Solomon GS (2000) Olfactory dysfunction discriminates probable Alzheimer's dementia from major depression: A cross-validation and extension. J Neuropsychiatry Clin Neurosci 12: 29-33.

29. Solomon GS, Petrie WM, Hart JR, Brackin HB Jr (1998) OIfactory dysfunction discriminates Alzheimer's dementia from major depression. J Neuropsychiatry Clin Neurosci 10: 64-67.

30. Negoias S, Hummel T, Symmank A, Schellong J, Joraschky $\mathrm{P}$, et al. (2016) Olfactory bulb volume predicts therapeutic outcome in major depression disorder. Brain Imaging Behav 10: 367-372.

31. Hummel T, Rissom K, Reden J, Hähner A, Weidenbecher $\mathrm{M}$, et al. (2009) Effects of olfactory training in patients with olfactory loss. Laryngoscope 119: 496-499.

32. Birte-Antina W, Ilona C, Antje H, Thomas H (2018) Olfactory training with older people. Int J Geriatr Psychiatry 33: 212-220.

33. Anderson RJ, Frye MA, Abulseoud OA, Lee KH, McGillivray JA, et al. (2012) Deep brain stimulation for treatment-resistant depression: Efficacy, safety and mechanisms of action. Neurosci Biobehav Rev 36: 1920-1933.

34. Mayberg HS, Lozano AM, Voon V, McNeely HE, Seminowicz D, et al. (2005) Deep brain stimulation for treatment-resistant depression. Neuron 45: 651-660.

35. Gottfried JA (2006) Smell: Central nervous processing. Adv Otorhinolaryngol 63: 44-69.

36. Atanasova B, Graux J, El Hage W, Hommet C, Camus V, et al. (2008) Olfaction: A potential cognitive marker of psychiatric disorders. Neurosci Biobehav Rev 32: 1315-1325.

37. Cumming AG, Matthews NL, Park S (2011) Olfactory identification and preference in bipolar disorder and schizophrenia. Eur Arch Psychiatry Clin Neurosci 261: 251-259.

38. Lombion-Pouthier S, Vandel P, Nezelof S, Haffen E, Millot $\mathrm{JL}$ (2006) Odor perception in patients with mood disorders. $\mathrm{J}$ Affect Disord 90: 187-191. 